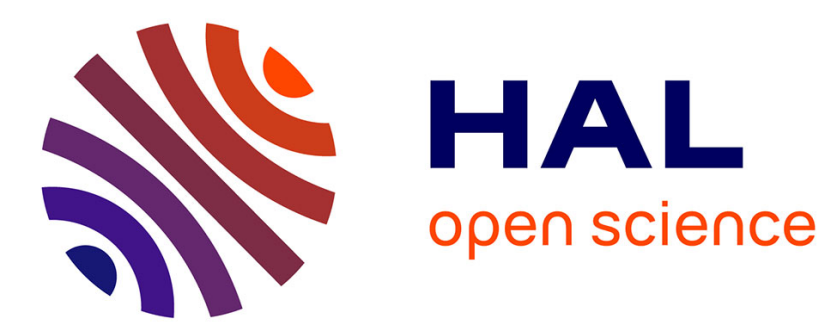

\title{
Effective viscosity of a random mixture of fluids
}

Benoit Noetinger, Laurène Hume, Robin Chatelin, Philippe Poncet

\section{To cite this version:}

Benoit Noetinger, Laurène Hume, Robin Chatelin, Philippe Poncet. Effective viscosity of a random mixture of fluids. Physical Review Fluids, 2018, 3 (1), pp.014103. 10.1103/PhysRevFluids.3.014103 . hal-01847035

\section{HAL Id: hal-01847035 \\ https://hal-ifp.archives-ouvertes.fr/hal-01847035}

Submitted on 23 Jul 2018

HAL is a multi-disciplinary open access archive for the deposit and dissemination of scientific research documents, whether they are published or not. The documents may come from teaching and research institutions in France or abroad, or from public or private research centers.
L'archive ouverte pluridisciplinaire HAL, est destinée au dépôt et à la diffusion de documents scientifiques de niveau recherche, publiés ou non, émanant des établissements d'enseignement et de recherche français ou étrangers, des laboratoires publics ou privés. 


\title{
Effective viscosity of a random mixture of fluids
}

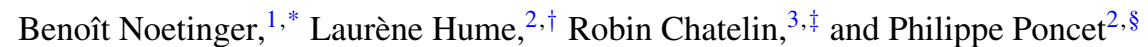

${ }^{1}$ IFPEN 1, 4 avenue de Bois Préau 92852 Rueil-Malmaison, France

${ }^{2}$ University Pau \& Pays Adour, LMAP, UMR CNRS 5142, IPRA, avenue de l'Université, F-64013 Pau, France

${ }^{3}$ Université de Lyon, ENISE, CNRS, UMR 5513, Laboratoire de Tribologie et Dynamique des Systèmes, 58 rue Jean Parot, 42023 Saint-Etienne Cedex 02, France

(1)

(Received 14 April 2017; published xxxxxx)

We propose an estimation of the effective viscosity of a random mixture of Newtonian fluids that ignores capillary effects. The local viscosity of the mixture is assumed to be a random function of the position. Using perturbation expansions up to the second order, the resulting formula can be recast under the form of a simple power averaging mixing low. Numerical tests are used to assess the validity of the formula and the range of its applicability.
\end{abstract}

DOI: 10.1103/PhysRevFluids.00.004100

Computing the effective viscosity of complex fluids such as suspensions and emulsions is an old problem studied by Einstein and Taylor [1,2] among many others. Such calculations have applications in rheology and related areas. The general issue is to find the connection between the large-scale rheological behavior, mainly the viscosity of the mixture, and information regarding the fluid microstructure. In the case of suspensions, these data can be the shapes, sizes, volume fractions, and pair correlation functions of the suspended particles [3-5]. Similar approaches have been used for emulsions and immiscible inclusions of one fluid into another [2,6]. In another context, closer to thermodynamics, the viscosity of gas or liquid mixtures can be estimated from statistical mechanics principles using the properties of each fluid and microscopic interaction parameters. Many formulas have been proposed to estimate the viscosity (see Ref. [7] and references therein). In the oil industry, glycerol-water mixtures have been studied experimentally in the context of oil refining [8,9], leading to the so-called "quarter power mixing rule":

$$
\eta_{\mathrm{eff}}=\left\langle\eta^{-1 / 4}\right\rangle^{-4}=\left[c \eta_{\text {Glycerol }}^{-1 / 4}+(1-c) \eta_{\mathrm{Water}}^{-1 / 4}\right]^{-4} .
$$

so In this equation, $\eta_{\text {eff }}$ is the effective viscosity of the mixture, $\eta_{\text {Glycerol }}$ and $\eta_{\text {Water }}$ are the respective 31 viscosities of pure fluids, and $c$ is the glycerol concentration; $\langle\cdot\rangle$ is the arithmetic averaging. To the best of our knowledge, these empirical observations do not have any theoretical underpinning.

In order to improve our understanding of viscosity homogenization, we will follow here the so-called stochastic approach [10-15], which is popular in the fields of random and composite materials and in hydrogeology. The key idea is to consider that the mixture can be represented by a single fluid characterized by a local viscosity modeled by a stationary random function which depends on the position. This random function is described by its two first moments: its local average

\footnotetext{
*benoit.noetinger@ifpen.fr

†laurene.hume@univ-pau.fr

${ }^{\ddagger}$ robin.chatelin@enise.fr

§philippe.poncet@univ-pau.fr
} 
and its two-point correlation function. Such approaches are still the subject of active investigations in the study of random materials when determining both the effective Young modulus and the electrical conductivity [13-16] and references therein. Related mathematical techniques range from homogenization and stochastic perturbation techniques to diagrammatic techniques of theoretical physics. Due to the formal analogies in the underlying equations, such approaches were also followed to study the permeability of heterogeneous rocks [10-12,14,17-20]. As far as we know, stochastic homogenization has never been carried out for viscosity. This is probably due to the emphasis on the study of suspensions and emulsions which require specific techniques, because most of the analytical difficulty comes from the boundary condition at the interface separating both fluids. In these systems, there is a strong focus on the relationship between the microstructure of the suspension and its time evolution which can lead to quite subtle organizational effects [21-23].

It seems rather difficult to study and set up real systems with random viscosity. First, emulsions with low surface tension seem to be good prospects. Indeed, the low surface tension hypothesis ensures that the pressure jump at the bubbles' interfaces which maintains their sphericity does not change the analysis. Otherwise a Taylor-like calculation accounting for the details of the flow inside and outside a single bubble $[2,6]$ is better suited.

Moreover, a variable viscosity Stokes model is typical of biomicrofluidics with heterogeneities. A second example is shear-thinning fluids exhibiting space-variable concentrations [24] $C$ in a globally constant shear fluid for which the Stokes equations are a good model as a first approximation (such as mucus or blood plasma). This leads to a viscosity $\mu(C, D)$ whose fluctuation correlation length remains statistically isotropic over time ( $D$ denotes the strain rate).

A third candidate could be a system with random temperature fields inducing in turn viscosity variations appearing as random. In cases in which the thermal diffusion coefficient is smaller than the dynamic viscosity associated with the momentum diffusion, the predictions of present work could be tested experimentally. Finally, in the widely studied case of suspensions, at a given scale the fluctuations of averaged volume fraction of the suspended particles also induces fluctuations of the local effective viscosity of the suspension. These fluctuations have an overall effect that can be studied following our approach. So in turn, these fluctuations can be related to the overall effective viscosity of the mixture. The present work could furthermore contribute to our understanding of fluctuation effects in suspensions, which are known to be of great importance (see Ref. [25] and references therein). Another goal is to justify the emergence of the quarter power mixing rule. In short the present study provides a quantification of any fluctuating effect on viscosity as long as the one-phase Newtonian model is acceptable. In addition, our result can provide an analytic benchmark to test numerical Stokes solvers, as is done in random porous media [26].

The paper is organized as follows: in Sec. II we present the Stokes equation and notations. In Sec. III we describe the random viscosity model. The definition of the effective viscosity is given in Sec. IV. In Sec. V A the perturbation method is carried out by means of a fluctuation expansion of the Stokeslet, as a power series of the viscosity fluctuations. Averaged results up to the second order are presented in Sec. VB. In Sec. VI we present the numerical methodology and results, followed by concluding remarks in Sec. VII.

\section{BASIC HYPOTHESIS AND EQUATIONS}

\section{A. Basic equations and notations}

We consider the flow of an incompressible Newtonian fluid in an infinite three-dimensional (3D) domain in the context of low Reynolds number hydrodynamics. The fluid's motion is governed by Stokes equations describing momentum and mass conservation:

$$
\begin{array}{r}
\nabla \cdot \tau(\mathbf{r})+\mathbf{f}(\mathbf{r})-\nabla p(\mathbf{r})=0, \\
\tau(\mathbf{r})=\eta(\mathbf{r})\left\{\nabla \mathbf{v}(\mathbf{r})+[\nabla \mathbf{v}(\mathbf{r})]^{t}\right\}, \\
\nabla \cdot \mathbf{v}(\mathbf{r})=0,
\end{array}
$$


83 where $\mathbf{r}, \mathbf{v}(\mathbf{r}), \eta(\mathbf{r})$, and $\tau(\mathbf{r})$ denote spatial position vector, the local fluid velocity, the local viscosity, 84 and the local viscous stress tensor, respectively. The quantities $\mathbf{f}(\mathbf{r})$ and $p(\mathbf{r})$ denote an arbitrary body 85 force field and the associated pressure, respectively. The boundary conditions at infinity are implicitly ${ }_{86}$ assumed to be $\mathbf{v}(\mathbf{r})=0, p(\mathbf{r})=0$, if the force field $\mathbf{f}(\mathbf{r})$ decays sufficiently fast.

$88 \quad$ In this section we consider that the viscosity is uniform: $\eta(\mathbf{r})=\eta_{0}$. Due to the underlying linearity 89 of the Stokes problem we can write

$$
\begin{aligned}
& \mathbf{v}(\mathbf{r})=\int d^{3} \mathbf{r}^{\prime} \mathbf{G}\left(\mathbf{r}-\mathbf{r}^{\prime}\right) \cdot \mathbf{f}\left(\mathbf{r}^{\prime}\right), \\
& p(\mathbf{r})=\int d^{3} \mathbf{r}^{\prime} \mathbf{P}\left(\mathbf{r}-\mathbf{r}^{\prime}\right) \cdot \mathbf{f}\left(\mathbf{r}^{\prime}\right) .
\end{aligned}
$$

90 The second order tensor $\mathbf{G}(\mathbf{r})$ is known as the Oseen tensor and is given by $\mathbf{G}(\mathbf{r})=\frac{1}{8 \pi \eta_{0}}\left(\frac{\mathbf{1}}{|\mathbf{r}|}+\frac{\mathbf{r r}}{|\mathbf{r}|^{3}}\right)$.

${ }_{91}$ Standard bold notations are used for vectors. The first order tensor $\mathbf{P}(\mathbf{r})=\frac{\mathbf{r}}{4 \pi|\mathbf{r}|^{3}}$ is the pressure tensor.

92 The convolution form suggests using Fourier transforms defined by

$$
h(\mathbf{q})=\int d^{3} \mathbf{r} e^{i \mathbf{q} \cdot \mathbf{r}} h(\mathbf{r})
$$

${ }_{93}$ This yields simpler linear relations between the Fourier transforms:

$$
\begin{aligned}
& \mathbf{v}(\mathbf{q})=\mathbf{G}(\mathbf{q}) \cdot \mathbf{f}(\mathbf{q}), \\
& p(\mathbf{q})=\mathbf{P}(\mathbf{q}) \cdot \mathbf{f}(\mathbf{q}),
\end{aligned}
$$

94 where

$$
\begin{gathered}
\mathbf{G}(\mathbf{q})=\frac{(\mathbf{1}-\hat{\mathbf{q}} \hat{\mathbf{q}})}{\eta_{0} q^{2}}, \\
\mathbf{P}(\mathbf{q})=\frac{i \mathbf{q}}{q^{2}} .
\end{gathered}
$$

${ }_{95}$ Here $\hat{\mathbf{q}}=\frac{\mathbf{q}}{q}$ is the unit vector built with $\mathbf{q}$. Using components we get more explicit expressions:

$$
\begin{gathered}
G_{\alpha \beta}(\mathbf{q})=\frac{\delta_{\alpha \beta}-\hat{q}_{\alpha} \hat{q}_{\beta}}{\eta_{0} q^{2}}, \\
P_{\alpha}(\mathbf{q})=\frac{i q_{\alpha}}{q^{2}} .
\end{gathered}
$$

96 When there is no ambiguity $\mathbf{r}$ and $\mathbf{q}$ will denote the real and Fourier variables, respectively.

\section{THE RANDOM MIXTURE}

In this section, we use the stochastic approach to introduce the random mixture model. The idea is to consider the local viscosity as being a random variable given by

$$
\eta(\mathbf{r})=\eta_{0}+\delta \eta(\mathbf{r})
$$

100 where $\eta_{0}$ is the arithmetic mean viscosity. The quantity $\delta \eta(\mathbf{r})$ is assumed to be a stationary random

101 variable, depending on the location $\mathbf{r}$, with the following properties:

$$
\begin{aligned}
\langle\eta(\mathbf{r})\rangle & =0, \\
\left\langle\delta \eta(\mathbf{r}) \delta \eta\left(\mathbf{r}^{\prime}\right)\right\rangle & =C\left(\mathbf{r}^{\prime}-\mathbf{r}\right) .
\end{aligned}
$$


102

The brackets $\langle\cdot\rangle$ represent the averaging over all possible realizations. The pair correlation function $C\left(\mathbf{r}^{\prime}-\mathbf{r}\right)$ of the viscosity fluctuation describes the spatial correlation between fluctuations at two points. For $\mathbf{r}^{\prime}-\mathbf{r}=\mathbf{0}, C(\mathbf{0})$ is the local variance of the viscosity. In order to have a well-defined Fourier transform $C(\mathbf{q})$ we assume that $C\left(\mathbf{r}^{\prime}-\mathbf{r}\right)$ decays sufficiently fast at infinity. In the isotropic case, the correlation function depends only on the modulus of $\left|\mathbf{r}^{\prime}-\mathbf{r}\right|$, so we have $C\left(\mathbf{r}^{\prime}-\mathbf{r}\right)=$ $C\left(\left|\mathbf{r}^{\prime}-\mathbf{r}\right|\right)$. We also have $C(\mathbf{q})=C(q)$ for the associated Fourier transform. In the simplest model, $\delta \eta(\mathbf{r})$ is assumed to be a multi-Gaussian variable. There are standard and efficient algorithms [27] to generate 3D maps of $\delta \eta(\mathbf{r})$ for an arbitrary covariance function $C(\mathbf{r})$ of the spatial range $l_{c}$. In the sequel we will consider an isotropic two-point correlation function depending only on the modulus of the lag vector $\left(\mathbf{r}^{\prime}-\mathbf{r}\right)$.

If we are not careful the viscosity can become negative in Eq. (11) because the noise $\delta \eta(\mathbf{r})$ has a multi-Gaussian distribution. In order to avoid this problem, we consider that the logarithm $\log \eta$ of the local viscosity follows a normal distribution, i.e., $\eta$ follows a log-normal distribution, which ensures positiveness. Therefore $\eta(\mathbf{r})=\eta_{g} \exp [\sigma Z(\mathbf{r})]$ where the quantities $\eta_{g}$ and $\sigma^{2}$ are the geometric average of the viscosity and the variance of the logarithmic viscosity, respectively. The quantity $Z(\mathbf{r})$ is a random function of position assumed to be multi-Gaussian and having a unit variance. Moreover $\sigma^{2}$ is dimensionless. This gives a nonambiguous mathematical meaning to a small variance.

\section{DEFINITION OF THE EFFECTIVE VISCOSITY}

In this section, we define the effective viscosity $\eta_{\text {eff }}$ of the random mixture. We follow a technique that was proposed in the study of suspensions [28,29] or random porous media [17].

Let $\mathbf{f}(\mathbf{r})$ be an arbitrary body force field acting on the fluid. In the case of a homogeneous fluid of viscosity $\eta_{\mathrm{eff}}$, one gets, using Fourier transforms:

$$
\begin{gathered}
\mathbf{v}(\mathbf{q})=\frac{(\mathbf{1}-\hat{\mathbf{q}} \hat{\mathbf{q}})}{\eta_{\mathrm{eff}} q^{2}} \cdot \mathbf{f}(\mathbf{q}), \\
p(\mathbf{q})=\frac{i \mathbf{q}}{q^{2}} \cdot \mathbf{f}(\mathbf{q}) .
\end{gathered}
$$

In the case of random mixture we expect after averaging a linear response of the form

$$
\begin{array}{r}
\langle\mathbf{v}(\mathbf{q})\rangle=\frac{(\mathbf{1}-\hat{\mathbf{q}} \hat{\mathbf{q}})}{\eta_{\mathrm{eff}}(q) q^{2}} \cdot \mathbf{f}(\mathbf{q}), \\
\langle p(\mathbf{q})\rangle=p(q) \frac{i \mathbf{q}}{q^{2}} \cdot \mathbf{f}(\mathbf{q}) .
\end{array}
$$

Such a form can be expected due to the linearity of the Stokes equations and to the statistical translational invariance of the system that leads to a convolution form after averaging. The second order calculation, presented in Sec. V, confirms this point. The so-called effective or equivalent fluid viscosity corresponds to the limit of these equations when $q$ tends to 0 . An algebraic definition of $\eta_{\text {eff }}$ can thus be proposed, if the limit exists:

$$
\eta_{\mathrm{eff}}=\lim _{q \rightarrow 0} \eta_{\mathrm{eff}}(q)
$$

By analogy with random porous media [17] and using the isotropy of the system, we can rigorously predict the equality $\langle p(q)\rangle \doteq 1$. This can be justified using arbitrary force fields derived from a potential: we consider a force field $\mathbf{f}(\mathbf{r})=-\nabla \phi(\mathbf{r})$, where the force potential $\phi(\mathbf{r})$ is arbitrary with Fourier transform $\phi(\mathbf{q})$. In this case one obtains $\mathbf{v}(\mathbf{q})=\mathbf{0}$ and $p(\mathbf{q})=\phi(\mathbf{q})$. As this equality is valid for any $\phi(\mathbf{q})$, we deduce that $p(\mathbf{q})=1$. This means that the average pressure tensor remains equal to its homogeneous counterpart after averaging. In other words, the role of pressure is to project the equation on divergence-free fields. This can be better understood by writing Eqs. (16) in the physical 
137 space:

$$
-\int d^{3} \mathbf{r}^{\prime} \eta_{\mathrm{eff}}\left(\left|\mathbf{r}^{\prime}-\mathbf{r}\right|\right) \nabla^{2}\left\langle\mathbf{v}\left(\mathbf{r}^{\prime}\right)\right\rangle=\mathbf{f}(\mathbf{r})-\nabla\langle p(\mathbf{r})\rangle, \quad \nabla \cdot\langle\mathbf{v}(\mathbf{r})\rangle=0 .
$$

138

which may be rewritten using the tensorial components:

$$
\mathbf{v}_{\alpha}^{n+1}(\mathbf{r})=-\varepsilon \int d^{3} \mathbf{r}^{\prime} \mathbf{G}_{\alpha \beta}\left(\mathbf{r}-\mathbf{r}^{\prime}\right) \partial_{\nu_{1}}\left\{\delta \eta\left(\mathbf{r}^{\prime}\right)\left[\partial_{\nu_{1}} \mathbf{v}_{\beta}^{n}\left(\mathbf{r}^{\prime}\right)+\partial_{\beta} \mathbf{v}_{\nu_{1}}^{n}\left(\mathbf{r}^{\prime}\right)\right]\right\} .
$$


170

Here $\partial_{x, y, \text { or } z}$ indicate spatial derivations with respect to the space variable $x, y, z$ as well as the variable if there is some ambiguity. The Einstein summation convention over repeated indices is fully satisfied. Finally, using integration by parts, one obtains

$$
\mathbf{v}_{\alpha}^{n+1}(\mathbf{r})=-\varepsilon \int d^{3} \mathbf{r}^{\prime}\left[\partial_{\nu_{1} r^{\prime}} \mathbf{G}_{\alpha \beta}\left(\mathbf{r}-\mathbf{r}^{\prime}\right)\right]\left\{\delta \eta\left(\mathbf{r}^{\prime}\right)\left[\partial_{\nu_{1}} \mathbf{v}_{\beta}^{n}\left(\mathbf{r}^{\prime}\right)+\partial_{\beta} \mathbf{v}_{\nu_{1}}^{n}\left(\mathbf{r}^{\prime}\right)\right]\right\} .
$$

73 This formula shows that the $n$th order term involves rather complex integrations of products of $n$ viscosity fluctuations evaluated at $n$ different points. After averaging, the first order term will vanish. 75 Combined with cumulant expansions of the $n$th order correlation functions of the viscosity, this 76 formula is the starting point of diagrammatic expansions that are beyond the scope of this paper. The rest of the paper is limited to second order term $(n=2)$.

\section{B. Second order results}

In this section, we compute the second order correction obtained after two applications of the recursion equation Eq. (25):

$$
\begin{aligned}
\mathbf{v}_{\alpha}^{2}(\mathbf{r})= & \varepsilon^{2} \int d^{3} \mathbf{r}_{1} \int d^{3} \mathbf{r}_{2}\left[\partial_{\nu_{1} r_{1}} \mathbf{G}_{\alpha \beta}\left(\mathbf{r}-\mathbf{r}_{1}\right)\right] \delta \eta\left(\mathbf{r}_{1}\right)\left\{\left[\partial_{\nu_{1} r_{1}} \partial_{\nu_{2} r_{2}} \mathbf{G}_{\beta \gamma}\left(\mathbf{r}_{1}-\mathbf{r}_{2}\right)\right] \delta \eta\left(\mathbf{r}_{2}\right)\right. \\
& \left.\times\left[\partial_{\nu_{2}} \mathbf{v}_{\gamma}^{0}\left(\mathbf{r}_{2}\right)+\partial_{\gamma} \mathbf{v}_{\nu_{2}}^{0}\left(\mathbf{r}_{2}\right)\right]+\left[\partial_{\beta r_{1}} \partial_{\nu_{2} r_{2}} \mathbf{G}_{v_{1} \gamma}\left(\mathbf{r}_{1}-\mathbf{r}_{2}\right)\right] \delta \eta\left(\mathbf{r}_{2}\right)\left[\partial_{\nu_{2}} \mathbf{v}_{\gamma}^{0}\left(\mathbf{r}_{2}\right)+\partial_{\gamma} \mathbf{v}_{\nu_{2}}^{0}\left(\mathbf{r}_{2}\right)\right]\right\}
\end{aligned}
$$

Since $C\left(\mathbf{r}_{2}-\mathbf{r}_{1}\right)=\left\langle\delta \eta\left(\mathbf{r}_{1}\right) \delta \eta\left(\mathbf{r}_{2}\right)\right\rangle$, one obtains after averaging over the viscosity fluctuations:

$$
\begin{aligned}
\left\langle\mathbf{v}_{\alpha}^{2}(\mathbf{r})\right\rangle= & \varepsilon^{2} \int d^{3} \mathbf{r}_{1} \int d^{3} \mathbf{r}_{2}\left[\partial_{\nu_{1} r_{1}} \mathbf{G}_{\alpha \beta}\left(\mathbf{r}-\mathbf{r}_{1}\right)\right]\left\{\left[\partial_{\nu_{1} r_{1}} \partial_{\nu_{2} r_{2}} \mathbf{G}_{\beta \gamma}\left(\mathbf{r}_{1}-\mathbf{r}_{2}\right)\right] C\left(r_{2}-r_{1}\right)\right. \\
& \left.\times\left[\partial_{\nu_{2}} \mathbf{v}_{\gamma}^{0}\left(\mathbf{r}_{2}\right)+\partial_{\gamma} \mathbf{v}_{\nu_{2}}^{0}\left(\mathbf{r}_{2}\right)\right]+\left[\partial_{\beta r_{1}} \partial_{\nu_{2} r_{2}} \mathbf{G}_{\nu_{1} \gamma}\left(\mathbf{r}_{1}-\mathbf{r}_{2}\right)\right] C\left(r_{2}-r_{1}\right)\left[\partial_{\nu_{2}} \mathbf{v}_{\gamma}^{0}\left(\mathbf{r}_{2}\right)+\partial_{\gamma} \mathbf{v}_{\nu_{2}}^{0}\left(\mathbf{r}_{2}\right)\right]\right\} .
\end{aligned}
$$

Up to the second order, this equation describes the average flow modification due to pair correlations between two viscosity fluctuations. Due to the statistical homogeneity, the final result appears under the form of several convolution products which are written under the form of simple products in the Fourier domain:

$$
\begin{aligned}
\left\langle\mathbf{v}_{\alpha}^{2}(\mathbf{q})\right\rangle= & \varepsilon^{2} i q_{\nu_{1}} \frac{(\mathbf{1}-\hat{\mathbf{q}} \hat{\mathbf{q}})_{\alpha \beta}}{\eta_{0} q^{2}}\left\{H_{\nu_{1} \nu_{2} \beta \gamma}(\mathbf{q})\left[i q_{\nu_{2}} \frac{(\mathbf{1}-\hat{\mathbf{q}} \hat{\mathbf{q}})_{\gamma \delta}}{\eta_{0} q^{2}}+i q_{\gamma} \frac{(\mathbf{1}-\hat{\mathbf{q}} \hat{\mathbf{q}})_{\nu_{2} \delta}}{\eta_{0} q^{2}}\right] f_{\delta}(\mathbf{q})\right. \\
& \left.+H_{\beta \nu_{2} \nu_{1} \gamma}(\mathbf{q})\left[i q_{\nu_{2}} \frac{(\mathbf{1}-\hat{\mathbf{q}} \hat{\mathbf{q}})_{\gamma \delta}}{\eta_{0} q^{2}}+i q_{\gamma} \frac{(\mathbf{1}-\hat{\mathbf{q}} \hat{\mathbf{q}})_{\nu_{2} \delta}}{\eta_{0} q^{2}}\right] f_{\delta}(\mathbf{q})\right\} .
\end{aligned}
$$

In this equation the tensor $H_{i j k l}(\mathbf{r})$ is defined by

$$
H_{i j k l}(\mathbf{r})=\partial_{i} \partial_{j} G_{k l}(\mathbf{r}) C(\mathbf{r}),
$$

87 and its Fourier transform is given by

$$
H_{i j k l}(\mathbf{q})=\int d^{3} \mathbf{r} e^{i \mathbf{q} \cdot \mathbf{r}} H_{i j k l}(\mathbf{r}) .
$$

Using our definition of the effective viscosity Eq. (17), we are interested in the low $q(\mathbf{q} \rightarrow 0)$ behavior of Eq. (28) that is expected to be proportional to $1 / q^{2}$. This can be verified by direct inspection of factors involving $i \mathbf{q} \frac{(\mathbf{1}-\hat{\mathbf{q}} \hat{\mathbf{q}})}{\eta_{0} q^{2}}$ inside Eq. (28), assuming that $H_{i j k l}(q=0)$ has a finite value. Assuming that the correlation function decays sufficiently fast at infinity [meaning $\lim _{q \rightarrow 0} H_{i j k l}(\mathbf{q})=H_{i j k l}(\mathbf{q}=0)$ 
192 exists], we check that the low- $q$ behavior of Eq. (26) is the same as

$$
\begin{aligned}
\left\langle\mathbf{v}_{\alpha}^{2}(\mathbf{q})\right\rangle= & \varepsilon^{2} i q_{\nu_{1}} \frac{(1-\hat{\mathbf{q}} \hat{\mathbf{q}})_{\alpha \beta}}{\eta_{0} q^{2}}\left\{H_{\nu_{1} \nu_{2} \beta \gamma}(\mathbf{q}=0)\left[i q_{\nu_{2}} \frac{(\mathbf{1}-\hat{\mathbf{q}} \hat{\mathbf{q}})_{\gamma \delta}}{\eta_{0} q^{2}}+i q_{\gamma} \frac{(\mathbf{1}-\hat{\mathbf{q}} \hat{\mathbf{q}})_{\nu_{2} \delta}}{\eta_{0} q^{2}}\right] f_{\delta}(\mathbf{q})\right. \\
& \left.+H_{\beta \nu_{2} \nu_{1} \gamma}(\mathbf{q}=0)\left[i q_{\nu_{2}} \frac{(\mathbf{1}-\hat{\mathbf{q}} \hat{\mathbf{q}})_{\gamma \delta}}{\eta_{0} q^{2}}+i q_{\gamma} \frac{(\mathbf{1}-\hat{\mathbf{q}} \hat{\mathbf{q}})_{\nu_{2} \delta}}{\eta_{0} q^{2}}\right] f_{\delta}(\mathbf{q})\right\} .
\end{aligned}
$$

193

194

to simplify notations, we can now set $\varepsilon=1$.

In the last equation, the fourth rank tensor $\mathbf{H}(\mathbf{q}=\mathbf{0})$ is defined by

$$
H_{i j k l}(\mathbf{q}=0)=\int d^{3} \mathbf{r} \partial_{i} \partial_{j} G_{k l}(\mathbf{r}) C(\mathbf{r}) .
$$

195

This equality can be transformed using Parseval's identity:

$$
\begin{aligned}
H_{i j k l}(\mathbf{q}=0) & =\frac{1}{(2 \pi)^{3}} \int d^{3} \mathbf{q} i q_{i} i q_{j} \frac{(\mathbf{1}-\hat{\mathbf{q}} \hat{\mathbf{q}})_{k l}}{\eta_{0} q^{2}} C(\mathbf{q}) \\
& =\frac{1}{(2 \pi)^{3}} \int d^{3} \mathbf{q} i \hat{q}_{i} i \hat{q}_{j} \frac{(\mathbf{1}-\hat{\mathbf{q}} \hat{\mathbf{q}})_{k l}}{\eta_{0}} C(\mathbf{q}) .
\end{aligned}
$$

196 We show in the Appendix that in the isotropic case, the following equality holds:

$$
H_{i j k l}(\mathbf{q}=0)=\left[-\frac{4}{15} \delta_{i j} \delta_{k l}+\frac{1}{15}\left\{\delta_{i k} \delta_{j l}+\delta_{i l} \delta_{j k}\right\}\right] \frac{C(r=0)}{\eta_{0}} .
$$

197 The tensor contractions involved when inserting Eq. (32) in Eq. (30) are carried out in the Appendix. 198 The final second order correction to the Stokeslet is equal to

$$
\frac{2}{5} \frac{(\mathbf{1}-\hat{\mathbf{q}} \hat{\mathbf{q}})}{\eta_{0}^{2} q^{2}} \times \frac{C(r=0)}{\eta_{0}} .
$$

199 As expected, this is proportional to the Oseen tensor $\frac{1-\hat{q} \hat{q}}{q^{2}}$. This result is remarkable because it shows 200 that up to the second order, in the Fourier domain, the $\mathbf{q} \rightarrow 0$ limit of the second order correction 201 of $\eta_{\text {eff }}$ depends only on the local variance of the viscosity fluctuations, and not on the particulars of 202 the whole correlation function. With this form we can use the equivalent viscosity $\eta_{\text {eff }}$, Eq. (16) and 203 Eq. (17) to get by direct identification the formula

$$
\eta_{\mathrm{eff}}=\eta_{0}\left[1-\frac{2}{5} \frac{C(0)}{\eta_{0}^{2}}\right],
$$

204 which is valid up to the second order. It is now more convenient to return to logarithms using the 205 formula (36), which is exact for log-normal variables and which is derived in the Appendix:

$$
\left\langle\eta^{\omega}\right\rangle^{\frac{1}{\omega}}=\eta_{g}\left(e^{(\omega \sigma)^{2} / 2}\right)^{\frac{1}{\omega}}=\eta_{g} \exp \left(\frac{\omega \sigma^{2}}{2}\right) .
$$

206 In the general case, this formula relating first and second moments of a random variable and its 207 logarithm is still valid up to the second order of interest. Using the same formula, $\eta_{0}=\langle\eta\rangle=$ $208 \eta_{g} \exp \frac{\sigma^{2}}{2}$. Thus one obtains at the second order:

$$
\begin{aligned}
\eta_{\mathrm{eff}} & =\eta_{0}\left[1-\frac{2}{5} \frac{C(0)}{\eta_{0}^{2}}\right] \\
& =\eta_{g}\left[1+\frac{1}{2} \frac{C(0)}{\eta_{0}^{2}}-\frac{2}{5} \frac{C(0)}{\eta_{0}^{2}}\right] \\
& =\eta_{g}\left[1+\frac{1}{10} \frac{C(0)}{\eta_{0}^{2}}\right] .
\end{aligned}
$$


The result in Eq. (35) may then be recast under the more compact and robust form of a power averaging formula, sharing the same second order expansion with respect to the log-viscosity variance $\sigma^{2} \simeq \frac{C(0)}{\eta_{0}{ }^{2}}$ for small $\sigma^{2}$ :

$$
\eta_{\mathrm{eff}}=\left\langle\eta^{\omega}\right\rangle^{\frac{1}{\omega}}
$$

212 with $\omega=\frac{1}{5}$.

${ }_{213}$ We recall that strictly speaking, this result is valid only at the second order. In practice it can 214 be computed even for a large viscosity variance. Its theoretical validity domain is controlled by the 215 validity of the second order expansion, and by the robustness of the proposed compact expression.

\section{NUMERICAL TESTS}

In this section we present numerical evidence of the validity of Eq. (37) using a Stokes solver for viscous flows. This validation requires the generation of random viscosity maps and the solution to the Stokes equations with variable viscosity. These tests are difficult to carry out because our homogenization result is valid only for a small viscosity variance, and simulations in these conditions may yield noisy results. A compromise must then be found between an accurate signal-to-noise ratio (which means working with quite large $\sigma^{2}$ ) and a sufficiently small value of $\sigma$ (in which case we remain in the theoretical validity domain).

We now set up a work flow to compare the effective viscosity derived from the computed velocity field and the value given by Eq. (37). We consider a cubic domain $\Omega$ filled by a random mixture of viscosity $\eta$. Inside the domain, fluid flows periodically between the lateral faces $\left\{x=x_{\min }\right\},\left\{x=x_{\max }\right\},\left\{y=y_{\min }\right\}$, and $\left\{y=y_{\max }\right\}$ under a uniform force in the $x$ direction. On the horizontal faces $\left\{z=z_{\min }\right\}$ and $\left\{z=z_{\max }\right\}$, no-slip conditions are imposed for the velocity field $\mathbf{v}$ : $\mathbf{v}=0$.

The velocity field $\mathbf{v}$ satisfies the Stokes equations:

$$
-\operatorname{div}[2 \eta D(\mathbf{v})]=\mathbf{f}-\nabla p \text { in } \Omega,
$$

where $D(\mathbf{v})=\left(\nabla \mathbf{v}+\nabla \mathbf{v}^{T}\right) / 2$ is the strain rate tensor, $p$ is the pressure, and $\mathbf{f}$ is the external force. We also set the incompressibility condition $\nabla \cdot \mathbf{v}=0$ in $\Omega$ and periodic boundary conditions on lateral faces. We note that this flow corresponds to the Poiseuille flow in the constant viscosity case.

For a given vector field $\mathbf{u}$, we will denote by $\zeta(\mathbf{u})$ the solution of the Poisson equation $-\nabla^{2} \zeta=-\nabla \cdot \mathbf{u}$ in $\Omega$ with homogeneous Neumann boundary conditions, so that $\mathbb{P} \mathbf{u}=\mathbf{u}-\nabla \zeta$ is the projection on divergence-free fields, with no slip-through condition (i.e., no normal velocity) 
251 at boundaries. It has been shown [34,35] that introducing the sequence $\left\{\mathbf{v}_{k}^{*}\right\}$ satisfying

$$
-\eta \nabla^{2} \mathbf{v}_{k+1}^{*}=\mathbf{f}+\left[2 D\left(\mathbb{P} \mathbf{v}_{k}^{*}\right)+\left(\nabla \cdot \mathbf{v}_{k}^{*}\right) I d\right] \nabla \eta \text { in } \Omega
$$

252 leads to the solution $\mathbf{v}$ of Eq. (38) by means of $\mathbf{v}=\lim _{k \rightarrow \infty} \mathbb{P} \mathbf{v}_{k}^{*}$.

253

254

255

256

257

Boundary conditions for Eq. (39) at each iteration are $\mathbf{v}_{k+1}^{*}=\nabla \zeta\left(\mathbf{v}_{k}^{*}\right)$ on $\left\{z=z_{\min }\right\}$ and $\left\{z=z_{\max }\right\}$, and periodic conditions for other faces. Moreover, solving Eq. (39), a Poisson equation, requires only the use of straightforward finite-difference stencils and a fast Fourier transform (FFT) solver. This leads to a robust numerical method, well suited for variable viscosity flows even with fluid-structure interactions $[24,35,36]$.

Once this calculation is carried out and the velocity field is computed, the effective viscosity $\eta_{\text {eff }}$ of the mixture can be identified using the equivalent flow rate obtained solving the Poiseuille flow with constant viscosity $\eta_{\text {eff. }}$. If we solve analytically the Stokes equation with homogeneous viscosity:

$$
\eta_{\mathrm{eff}} \nabla^{2} \mathbf{v}_{\mathrm{eff}}=\mathbf{f} \text { in } \Omega \text {, }
$$

with the same boundary conditions as in Eq. (38), we can then estimate $\eta_{\text {eff }}$ by direct identification. The effective viscosity $\eta_{\text {eff }}$ is defined such that the velocity field $\mathbf{v}_{\text {eff }}$ solution to Eq. (40) has the same mean flow rate as the field $\mathbf{v}$ solution to Eq. (38). Given that the external force $\mathbf{f}$ has only an $x$ component, then using the boundary conditions, we can write $\mathbf{v}_{\text {eff }}=(v(z), 0,0)^{T}$. The analytical solution of the Stokes equation may be found in textbooks and yields

$$
\eta_{\mathrm{eff}}=\frac{\left(y_{\max }-y_{\min }\right) z_{\max }^{3}}{12 Q_{\mathrm{num}}} f,
$$

where the global flow rate $Q_{\text {num }}$ is computed from the numerical integration of the solution $\mathbf{v}$. The resulting effective viscosity $\eta_{\text {eff }}$ can be compared to the result given by Eq. (37).

In our computations, the values $x_{\min }, y_{\min }, z_{\min }$ are set to 0 and $x_{\max }, y_{\max }, z_{\max }$ are set to 1 , so that the domain is the unit cube. This cell is then discretized with a Cartesian grid having resolution $N^{3}$ ( $N$ points in each direction, $N=129,193$, or 257 ).

\section{B. Random field generation}

Random viscosity fields are built using a 3D version of FFT-MA correlated random field generator [27]. This algorithm produces random fields efficiently by taking advantage of the Fourier transform. In the algorithm's implementation, we first generate a symmetric normalized covariance field $C_{N}$, following the so-called Gaussian model:

$$
C_{N}(h)=\exp \left[-\left(\frac{h}{l_{c}}\right)^{2}\right],
$$

where $h$ is the space lag and $l_{c}$ is the correlation length. In what follows the length $l_{c}$ is always expressed with respect to the space step $\delta x$ of a given discretization grid for the unit cube. This characteristic length is then written in the form $n_{c} \delta x$, where $n_{c}$ is an integer. The associated variance is taken equal to unity. A Gaussian white noise $z$ is also generated, and the final random field can be written as

$$
Z(\mathbf{r})=Z_{0}+\mathcal{F}^{-1}\left[\sqrt{\mathcal{F}\left(C_{N}\right)} \mathcal{F}(z)(\mathbf{r})\right],
$$

where $\mathcal{F}$ (respectively $\mathcal{F}^{-1}$ ) is the discrete Fourier transform (respectively the discrete inverse Fourier transform) in the 3D space, and $Z_{0}$ is the arithmetic average of $Z$.

The final viscosity field is then given by

$$
\eta=\eta_{g} \exp \left\{\sigma \mathcal{F}^{-1}\left[\sqrt{\mathcal{F}\left(C_{N}\right)} \mathcal{F}(z)\right]\right\} .
$$

283 In the sequel, the quantity $\eta_{g}$ denotes the expected geometric average of $\eta$ and will be set to 1 . In 284 Fig. 1 three random fields are plotted with different correlation lengths $\left(l_{c}=3 \delta x, 10 \delta x\right.$, and $25 \delta x$ at 285 resolution $257^{3}$ ). Plotting the log viscosity histograms of one particular realization in Fig. 2 shows 

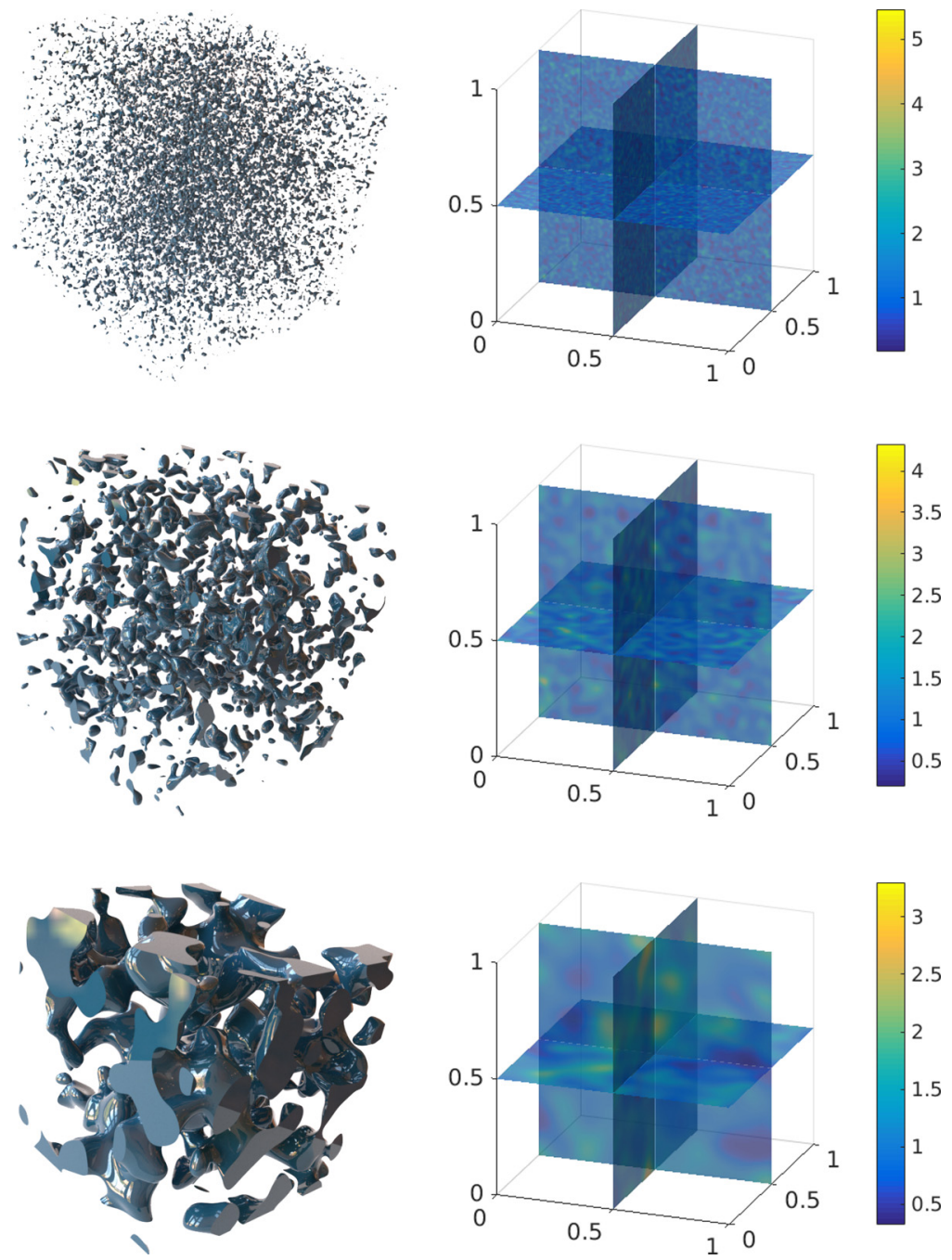

FIG. 1. Random fields using isolevel surfaces and slices of viscosity $\left(\sigma^{2}=0.1, l_{c}=3 \delta x, 10 \delta x\right.$, and $25 \delta x$ at resolution $257^{3}$, top to bottom).

that the histograms of viscosity logarithms are Gaussian. The small discrepancies may be explained by finite-size effects.

With this particular Fourier-based method, we can build periodic fields in the $x$ and $y$ directions in order to ensure consistency with boundary conditions of the reference Poiseuille problem and in order to avoid spurious discontinuities. In the $z$ direction, the presence of the nonslip conditions does not impose any $z$ periodicity. After the observation of results with periodic or nonslip conditions in this direction, the direct effect on the estimated effective viscosity is negligible. We recall that the different correlation lengths are expressed under the form $n_{c} \delta x$, where $n_{c}$ varies from 1 to $m, m$ being the greater integer such that $m \times d x \leqslant 0.04$. We also recall that the input viscosity geometric average is set to $\eta_{g}=1$.

Figure 3 shows expected and computed properties for 900 independent viscosity random fields generated by FFT-MA with resolution $257^{3}$. On the top plot, each point corresponds to a posteriori estimation of the geometric average using volume average of the corresponding viscosity map. We note that the computed average is then close to the input geometric mean (equal to 1). The dispersion 


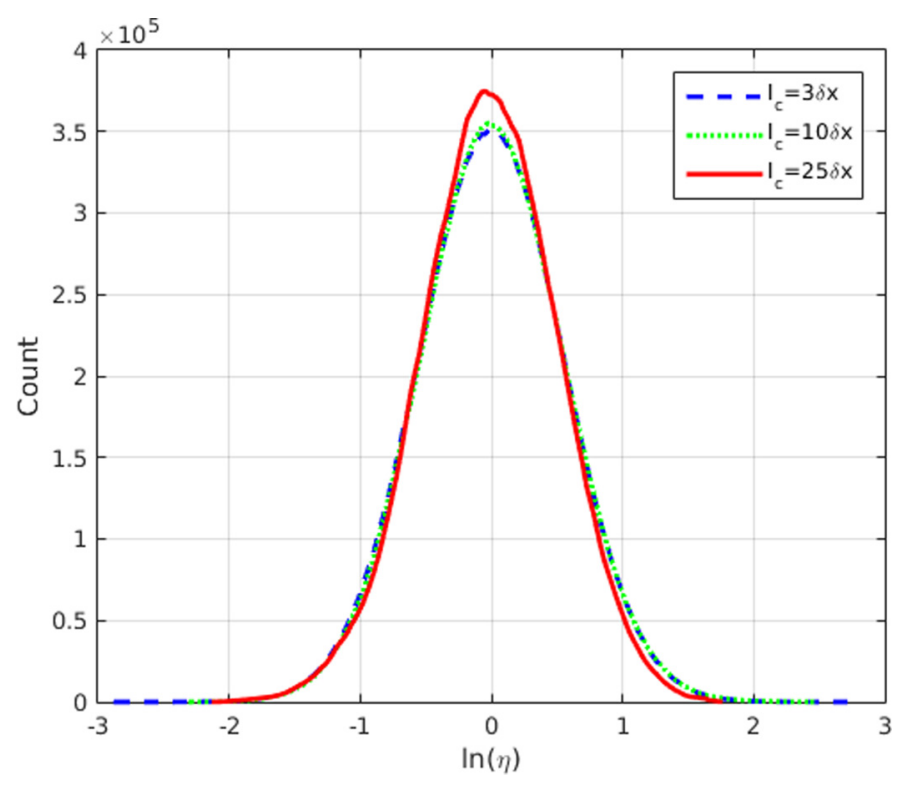

FIG. 2. Histograms of the values of $\ln (\eta)\left(\sigma^{2}=0.3\right)$ for three correlation lengths.

of the data depends strongly on the correlation length. We can expect such behavior due to the central limit theorem: the variance of the volume average scales as $\sigma^{2}\left(l_{c}{ }^{3} / V\right), l_{c} \ll V^{1 / 3}$ where $V$ is the volume of the unit cell $(V=1$ in present case). We also note the large scaling factor of the plot in Fig. 3. In order to conclude this section, the bottom graph in Fig. 3 presents the empirical log viscosity variance computed for each of the 900 fields versus the input imposed variance $\sigma$. We observe a perfect agreement as the correlation between observed and input variance is excellent.

\section{Interpretation methodology}

In the sequel we present the simulation results on the unit cell $\Omega$ discretized with 129,193 , and 257 grid points in each direction for both the log viscosity and the numerical solution of the Stokes equation. This discretization choice corresponds to space steps $\delta x=\delta y=\delta z$ going from $7.8 \times 10^{-3}$ to $3.9 \times 10^{-3}$.

In order to set up our comparisons, we still take advantage of the log-normality to rewrite the power average formula (37) with $\omega=1 / 5$ in the form

$$
\ln \left(\eta_{\mathrm{eff}}\right)=\frac{1}{5} \frac{\sigma^{2}}{2}
$$

This result shows that by plotting $\ln \left(\eta_{\mathrm{eff}}\right)$ with respect to $\sigma^{2} / 2$, a slope of $1 / 5$ is expected at least for small variances. We recall that our theoretical result (37) was obtained using averaging of the apparent viscosity over all the detailed viscosity realizations. In order to test this theoretical result, we have to compute the effective viscosity of several independent realizations of the input viscosity maps, and then average these values. The associated linear regression should then exhibit the 0.2 slope. For each realization, the effective viscosity $\eta_{\text {eff }}$ is computed with the expression (41).

We set up a Monte Carlo study by computing the effective viscosity of several independent viscosity realizations sharing the same covariance structure. Each experiment is conducted as follows: first, we generate a random viscosity field $\eta$ with FFT-MA (44) with unit geometric mean and expected variance $\sigma^{2}$; then we solve Stokes equations with variable viscosity field $\eta$; finally we compute $\ln \left(\eta_{\mathrm{eff}}\right)$ for this input viscosity field using Eq. (41). A linear regression is then performed using the resulting set of values $\ln \left(\eta_{\mathrm{eff}}\right)_{i}$ with respect to $\sigma_{i}^{2}$, where $i$ denotes the $i$ th experiment with the associated $i$ th viscosity random field. For each grid size and correlation length, 10 sets of viscosity random fields 

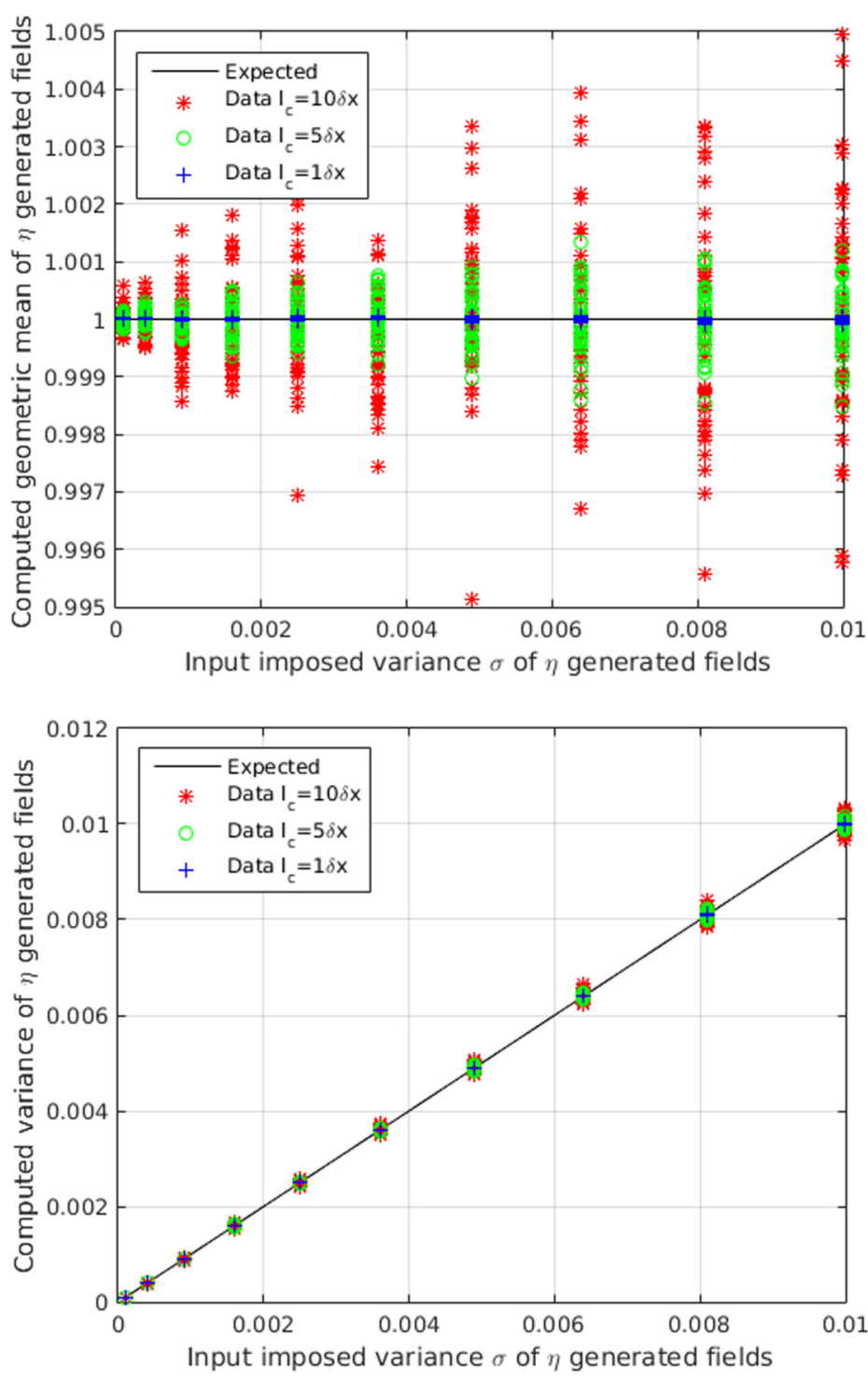

FIG. 3. Empirical and expected properties of generated $\eta$ fields.

326 are generated where the $i$ th field of a set corresponds to an input variance $\sigma^{2}=0.001 i$. In order

to get enough data to achieve the stabilization of linear regressions we generate between 10 and 40 independant sets. The number of generated sets for each grid resolution and $l_{c}$ value is displayed in Table I. For each value of $\sigma^{2} \in[0.001,0.01]$ we then obtain sets composed of 10 to 40 realizations.

For a given variance or correlation length, we generate additional sets and go on until the regression slope converges, with a maximum of 40 sets in total. We stop adding sets when the resulting slope is close to the initial one. This method leads to a massive data storage and the manipulation of many data files. For example the total size of all the viscosity fields generated for $257^{3}$ resolution represents more than $800 \mathrm{~GB}$.

\section{Results}

On Fig. 4 the effective $\log$ viscosity $\ln \left(\eta_{\text {eff }}\right)$ is plotted versus the real variance (real $\left.\sigma\right)^{2} / 2$ for two different correlation lengths in the case of the unit domain discretized by $257^{3}$ points. The obtained 
TABLE I. Number of realizations for each case.

\begin{tabular}{|c|c|c|c|c|c|c|c|c|c|c|c|c|}
\hline Resolution & & & $129^{3}$ & & & & & & $193^{3}$ & & & \\
\hline$l_{c} / \delta x$ & 1 & 2 & 3 & 4 & 5 & 1 & 2 & 3 & 4 & 5 & 6 & 7 \\
\hline No. sets & 40 & 40 & 40 & 40 & 40 & 20 & 20 & 20 & 40 & 40 & 40 & 40 \\
\hline Resolution & \multicolumn{12}{|c|}{$257^{3}$} \\
\hline$l_{c} / \delta x$ & 1 & 2 & 3 & 4 & 5 & 6 & 7 & 8 & 9 & 10 & & \\
\hline No. sets & 10 & 20 & 20 & 30 & 40 & 35 & 25 & 20 & 40 & 40 & & \\
\hline
\end{tabular}
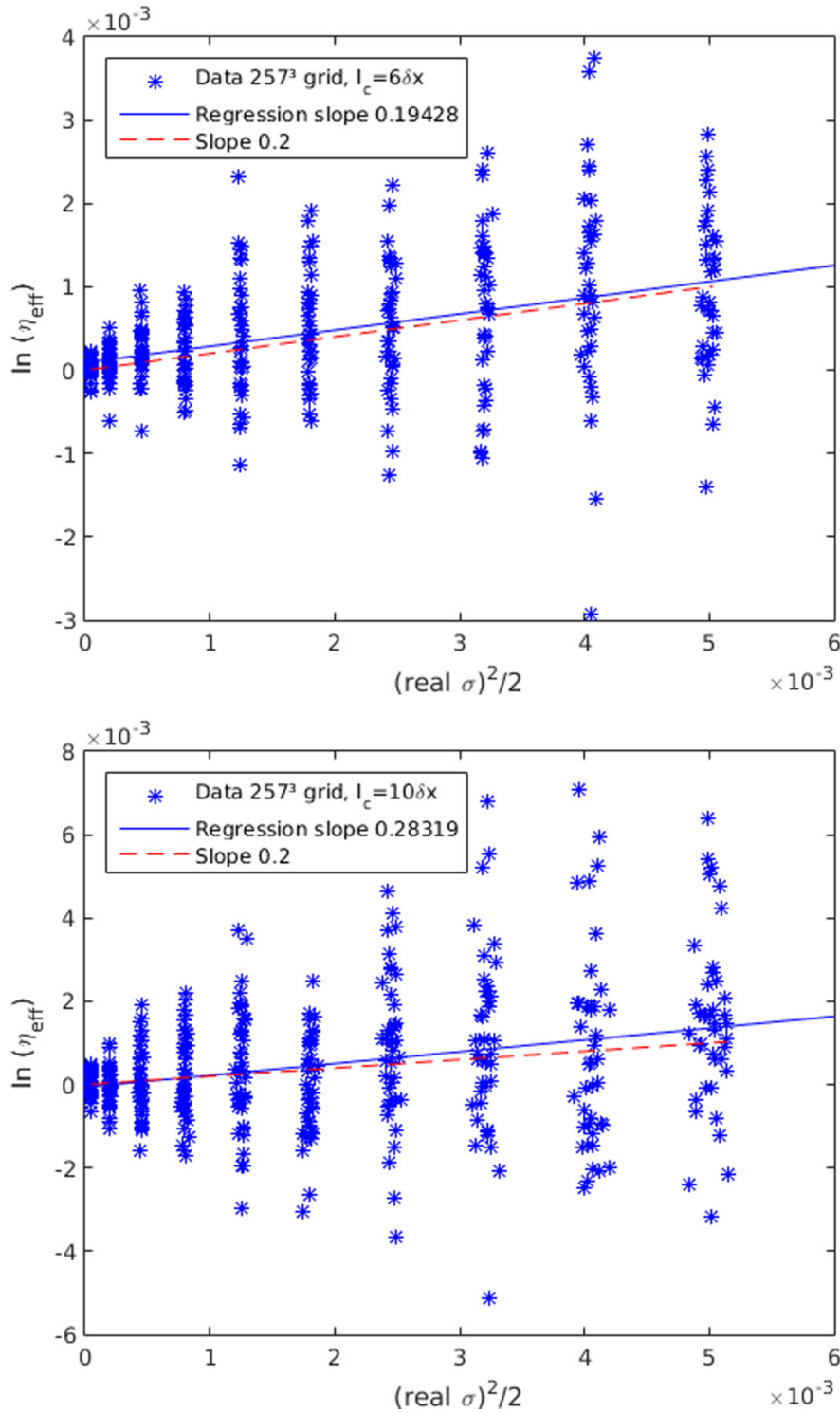

FIG. 4. Regressions for $l_{c}=6 \delta x$ and $10 \delta x$ on $257^{3}$ grid. 


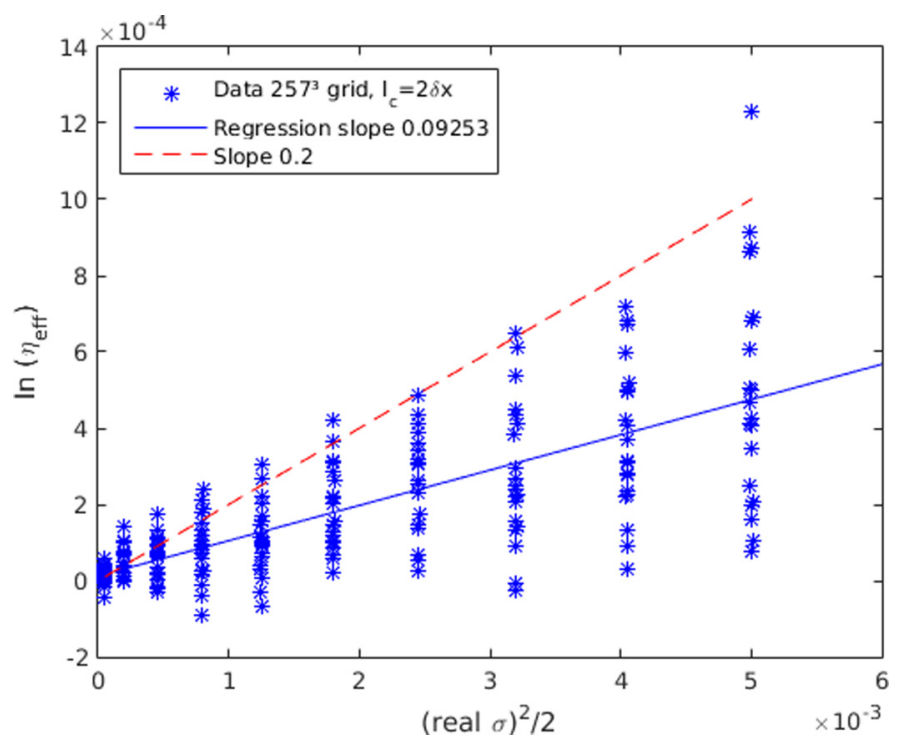

FIG. 5. Regressions for $l_{c}=2 \delta x$ on $257^{3}$ grid.

least square regression is plotted in dotted blue, and the expected theoretical straight line of slope 0.2 is plotted in plain red. As expected, the spreading of the data strongly depends on the correlation length (this was already the case for the geometric average plot) and the spreading due to the randomness of the underlying local viscosity fluctuations looks similar. A direct consequence of the data dispersion is that we need more independent realizations for high correlation lengths in order to get a reliable and stable regression slope. With a sufficient number of simulations, the theoretical slope $1 / 5$ remains in good agreement with computations in a range of correlation lengths which increases as the grid resolution increases. For a small correlation length, we display the corresponding data and linear regression in Fig. 5. The apparent averaging exponent is smaller than the theoretical prediction. This may be explained by a systematic bias introduced by the poor discretization. However, this observation is in good agreement with the results obtained by Ref. [26] in the case of conductivity and permeability. In the opposite case of large correlation lengths, a regression slope close to unity is expected. For the very large correlation lengths (compared to the overall system size), the viscosity map is in fact homogeneous. The viscosity $\eta_{\text {eff }}$ obtained by simulation will then be equal to the uniform common value, which remains a random log-normal variable (one single value per map). For other in-between correlation lengths, the apparent averaging exponent is expected to increase as the correlation length increases, with a plateau at 0.2 . Figures 4 and 5 show the regression plots, which highlight the averaging exponent for different $l_{c}$ at resolution $257^{3}$.

In Fig. 6 the averaging exponents given by through the regressions are plotted with respect to the correlation length for three grid resolutions. The curve starts from small negative values for uncorrelated media, and then reaches a plateau close to the theoretical value of $1 / 5$ before increasing to unity. We note that a very high resolution $\left(257^{3}\right)$ is needed to validate the theoretical result.

We can also observe that, for each regression, the data deviation from the regression line is of the form $\varepsilon \sqrt{(\text { real } \sigma)^{2} / 2}$ (see, e.g., Fig. 4). It is then possible to rewrite the regression formula in the form

$$
\ln \left(\eta_{e f f}\right)=p(\text { real } \sigma)^{2} / 2+b+\varepsilon \sqrt{(\text { real } \sigma)^{2} / 2},
$$

with $p$ the regression slope and $b$ the intercept. This is transformed into a multilinear expression which is used in the R software package. This provides the standard error estimation for slope. Error bars on the lower panel of Fig. 6 represent $95 \%$ confidence intervals with respect to this estimation. 

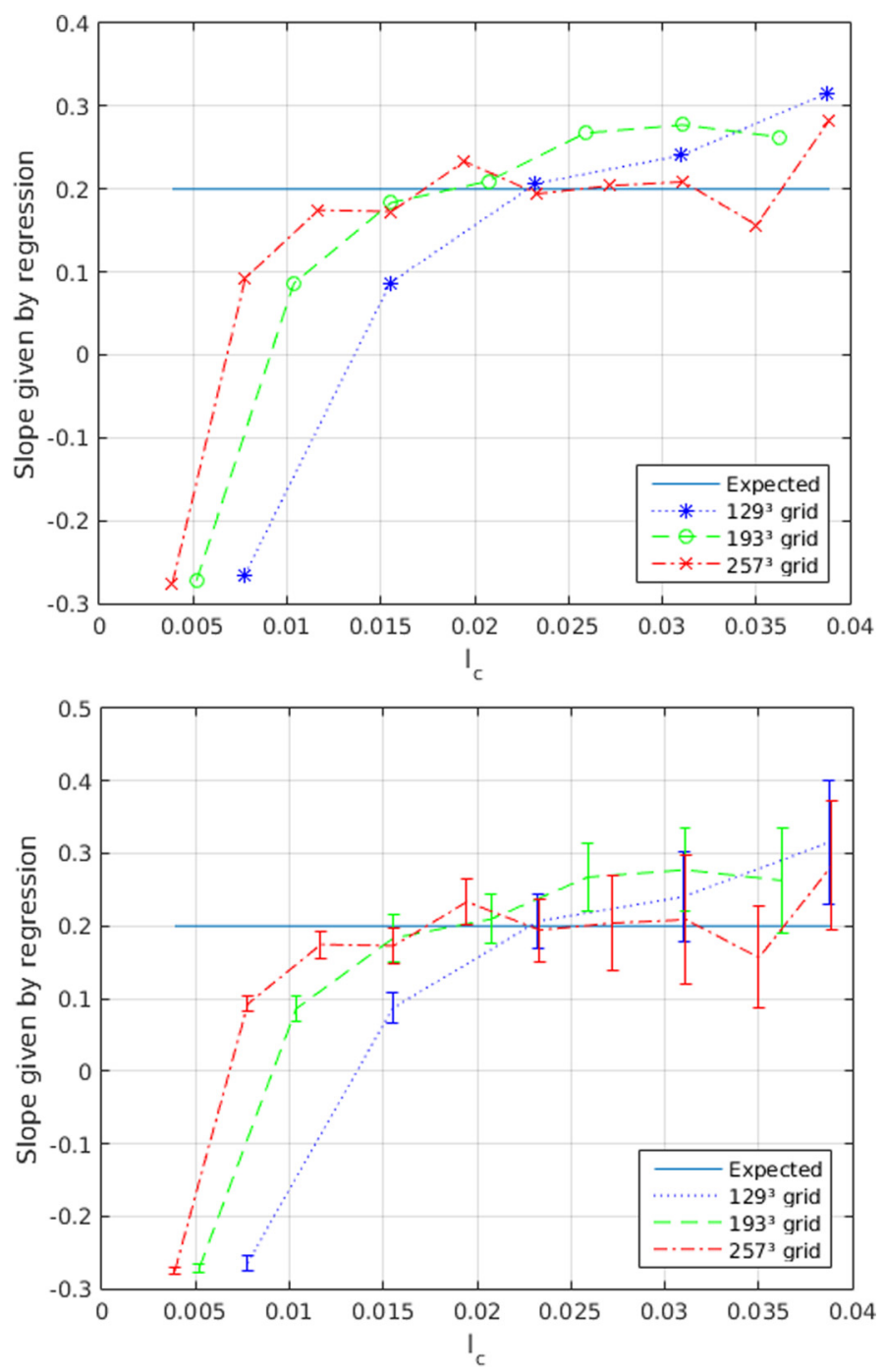

FIG. 6. Regression slopes of $\ln \left(\eta_{\text {eff }}\right)$ vs (real $\left.\sigma\right)^{2} / 2$ for different values of $l_{c}$ and resolutions. Top : slope estimations; bottom: slope estimations with $95 \%$ confidence intervals.

\section{COMMENTS AND CONCLUDING REMARKS}

Simulations provide results in good agreement with the power averaging formula (37), as the regression slopes present a plateau close to the theoretical value of 0.2 . For the two coarser grids of $129^{3}$ and $193^{3}$ resolutions, an inflection in the slopes can be observed around this value. In order to obtain significant results, both a sufficient number of discretization points per correlation length and many correlation lengths are necessary to ensure the stabilization of the effective viscosity. There is actually no generalized result on the convergence with respect to the grid resolution, although we can expect such converging behavior because of the central limit theorem.

The range of $\sigma^{2}$ for viscosity random fields has been carefully chosen. Small values of $\sigma^{2}$ under 0.001 lead to meaningless results because small numerical errors dominate when viscosity fluctuations are so small. In the same way, values greater than 0.01 must be considered with caution 
because the theory is based on the series expansion of $\sigma^{2}$. Furthermore, if we use low correlation lengths with $\sigma^{2} / 2>0.22$, the high values of $\nabla \eta$ can prevent the Stokes solver from converging.

We note that the variance of the observed effective viscosity depends strongly on the correlation length. This imposes severe constraints regarding the size of the computational grid.

In the case of the effective conductivity or permeability of random media, the power averaging formula (which is exact in one or two dimensions) is proved to be a very efficient approximation in three dimensions, up to log-conductivity variances of seven corresponding to variations of local conductivity of several orders of magnitude [30]. The results of this paper demonstrate that this approximation appears less robust in the context of the Stokes equation. A possible explanation is that due to its $1 / q^{2}$ factor, the Stokes equation propagator over-amplifies low frequency fluctuations. Our observation is also reminiscent of the observed wide fluctuations of sedimentation velocity in suspensions and the appearance of large structures (see Ref. [25] and references therein).

Finally, with reference to the known rheological literature, the power average formula with averaging power of $1 / 5$ does not compare well with analogous results as provided by the quarter power mixing rule $-1 / 4[2,6,8,9]$. We note that in the case of the effective viscosity of emulsions, the strong capillarity assumption which ensures overall sphericity of the bubbles changes the local boundary conditions of the flow at the bubbles' boundaries. As a result, an increased energy dissipation is expected, and thus a greater effective viscosity $[2,6]$ is obtained. This happens even if both fluids are assumed to share an almost common viscosity in order to be consistent with our calculations.

The effective viscosity averaging formula derived in this paper may be considered as a first estimator as far as minimal information is provided regarding the microstructure of a mixture. Improvement can be obtained if additional information becomes available. From the point of view of computational fluid dynamics (CFD), the proposed formula can be used to test numerical methods aiming at solving Stokes equations with variable viscosity. These numerical issues are the source of major progress toward the development of micro- and nanofluidics modeling tools.

\section{Evaluation of tensor $H$ in the isotropic case}

In the case of isotropic correlations, the following quantity must be computed in order to obtain explicit second order results for the effective viscosity:

$$
\begin{aligned}
H_{i j k l}(\mathbf{q}=0) & =\frac{1}{(2 \pi)^{3}} \int d^{3} \mathbf{q} i q_{i} i q_{j} \frac{(\mathbf{1}-\hat{\mathbf{q}} \hat{\mathbf{q}})_{k l}}{\eta_{0} q^{2}} C(q) \\
& =\frac{1}{(2 \pi)^{3}} \int_{0}^{\infty} 4 \pi C(q) q^{2} d q \frac{1}{4 \pi} \int d^{2} \hat{\mathbf{q}} i \hat{q}_{i} i \hat{q}_{j} \frac{(\mathbf{1}-\hat{\mathbf{q}} \hat{\mathbf{q}})_{k l}}{\eta_{0}} \\
& =C(r=0) \frac{1}{4 \pi} \int d^{2} \hat{\mathbf{q}} i \hat{q}_{i} i \hat{q}_{j} \frac{(\mathbf{1}-\hat{\mathbf{q}} \hat{\mathbf{q}})_{k l}}{\eta_{0}} .
\end{aligned}
$$

Due to the isotropy, the angular integral does not depend on $q$. The term $C(r=0)$ can then be recognized by computing the inverse Fourier transform. In order to compute the full tensor components, several integrals over the unit sphere must be computed:

$$
K_{i j k l}=\frac{1}{4 \pi} \int_{S_{1}} d^{2} \hat{\mathbf{q}} i \hat{q}_{i} i \hat{q}_{j}(\mathbf{1}-\hat{\mathbf{q}} \hat{\mathbf{q}})_{k l} .
$$

409 By isotropy, the integral of $\frac{1}{4 \pi} \int_{S_{1}} d^{2} \hat{\mathbf{q}} i \hat{q}_{i} i \hat{q}_{j}$ is proportional to the unit tensor, so it is sufficient to 410 evaluate its trace. We therefore obtain

$$
\frac{1}{4 \pi} \int_{S_{1}} d^{2} \hat{\mathbf{q}} i \hat{q}_{i} i \hat{q}_{j} \delta_{k l}=-\frac{1}{3} \delta_{i j} \delta_{k l} .
$$


${ }_{411}$ The same type of reasoning can be followed for the evaluation of the other term $\frac{1}{4 \pi} \int_{S_{1}} d^{2} \hat{\mathbf{q}} i \hat{q}_{i} i \hat{q}_{j} \hat{q}_{k} \hat{q}_{l}$, 412 which is obviously symmetric over all its pairs of indices. The expression is thus proportional to ${ }_{413}$ the tensor $\delta_{i j} \delta_{k l}+\delta_{i k} \delta_{j l}+\delta_{i l} \delta_{j k}$. The proportionality constant may be determined by evaluating its ${ }_{414}$ partial trace over any pair of indices. We finally obtain

$$
\frac{-1}{4 \pi} \int_{S_{1}} d^{2} \hat{\mathbf{q}} i \hat{q}_{i} i \hat{q}_{j} \hat{q}_{k} \hat{q}_{l}=\frac{1}{15}\left\{\delta_{i j} \delta_{k l}+\delta_{i k} \delta_{j l}+\delta_{i l} \delta_{j k}\right\} .
$$

415 Returning to tensor $\mathbf{H}$, we then obtain

$$
\begin{aligned}
H_{i j k l}(\mathbf{q}=0) & =-\left[\frac{1}{3} \delta_{i j} \delta_{k l}+\frac{1}{15}\left\{\delta_{i j} \delta_{k l}+\delta_{i k} \delta_{j l}+\delta_{i l} \delta_{j k}\right\}\right] \frac{C(r=0)}{\eta_{0}} \\
& =\left[-\frac{4}{15} \delta_{i j} \delta_{k l}+\frac{1}{15}\left\{\delta_{i k} \delta_{j l}+\delta_{i l} \delta_{j k}\right\}\right] \frac{C(r=0)}{\eta_{0}},
\end{aligned}
$$

416 which is Eq. (33).

425

We start from

$$
\begin{aligned}
i q_{\nu_{1}} & \frac{(\mathbf{1}-\hat{\mathbf{q}} \hat{\mathbf{q}})_{\alpha \beta}}{\eta_{0} q^{2}}\left\{\left[\frac{-4}{15} \delta_{\nu_{1} \nu_{2}} \delta_{\beta \gamma}+\frac{1}{15}\left(\delta_{\nu_{1} \beta} \delta_{\nu_{2} \gamma}+\delta_{\nu_{1} \gamma} \delta_{\nu_{2} \beta}\right)\right]\left[i q_{\nu_{2}} \frac{(\mathbf{1}-\hat{\mathbf{q}} \hat{\mathbf{q}})_{\gamma \delta}}{\eta_{0} q^{2}}+i q_{\gamma} \frac{(\mathbf{1}-\hat{\mathbf{q}} \hat{\mathbf{q}})_{\nu_{2} \delta}}{\eta_{0} q^{2}}\right]\right. \\
& \left.+\left[-\frac{4}{15} \delta_{\beta \nu_{2}} \delta_{\nu_{1} \gamma}+\frac{1}{15}\left(\delta_{\beta \nu_{1}} \delta_{\nu_{2} \gamma}+\delta_{\beta \gamma} \delta_{\nu_{2} \nu_{1}}\right)\right]\left[i q_{\nu_{2}} \frac{(\mathbf{1}-\hat{\mathbf{q}} \hat{\mathbf{q}})_{\gamma \delta}}{\eta_{0} q^{2}}+i q_{\gamma} \frac{(\mathbf{1}-\hat{\mathbf{q}} \hat{\mathbf{q}})_{\nu_{2} \delta}}{\eta_{0} q^{2}}\right]\right\} \\
= & i q_{\nu_{1}} \frac{(\mathbf{1}-\hat{\mathbf{q}} \hat{\mathbf{q}})_{\alpha \beta}}{\eta_{0} q^{2}}\left[-\frac{1}{5}\left(\delta_{\nu_{1} \nu_{2}} \delta_{\beta \gamma}+\delta_{\beta \nu_{2}} \delta_{\nu_{1} \gamma}\right)+\frac{2}{15} \delta_{\nu_{1} \beta} \delta_{\nu_{2} \gamma}\right]\left[i q_{\nu_{2}} \frac{(\mathbf{1}-\hat{\mathbf{q}} \hat{\mathbf{q}})_{\gamma \delta}}{\eta_{0} q^{2}}+i q_{\gamma} \frac{(\mathbf{1}-\hat{\mathbf{q}} \hat{\mathbf{q}})_{\nu_{2} \delta}}{\eta_{0} q^{2}}\right] \\
= & -\frac{1}{5}\left[i q_{\nu_{2}} \frac{(\mathbf{1}-\hat{\mathbf{q}} \hat{\mathbf{q}})_{\alpha \gamma}}{\eta_{0} q^{2}}+i q_{\gamma} \frac{(\mathbf{1}-\hat{\mathbf{q}} \hat{\mathbf{q}})_{\alpha \nu_{2}}}{\eta_{0} q^{2}}\right]\left[i q_{\nu_{2}} \frac{(\mathbf{1}-\hat{\mathbf{q}} \hat{\mathbf{q}})_{\gamma \delta}}{\eta_{0} q^{2}}+i q_{\gamma} \frac{(\mathbf{1}-\hat{\mathbf{q}} \hat{\mathbf{q}})_{\nu_{2} \delta}}{\eta_{0} q^{2}}\right] \\
= & \frac{2}{5} \frac{(\mathbf{1}-\hat{\mathbf{q}} \hat{\mathbf{q}})_{\alpha \delta}}{\eta_{0}^{2} q^{2}} .
\end{aligned}
$$

Throughout this calculation, we have removed the contraction with $f_{\delta}(\mathbf{q})$, which is of no interest.

\section{Power average of log-normal variables}

The general equality (36) may be derived using Gaussian integrals for log-normal variables:

$$
\left\langle\eta^{\omega}\right\rangle^{\frac{1}{\omega}}=\eta_{g}\left(e^{(\omega \sigma)^{2} / 2}\right)^{\frac{1}{\omega}}=\eta_{g} \exp \left(\frac{\omega \sigma^{2}}{2}\right) .
$$

$$
\left\langle\eta^{\omega}\right\rangle^{\frac{1}{\omega}}=\eta_{g}\langle\exp (\omega \sigma \zeta)\rangle^{\frac{1}{\omega}}
$$

426 with $\zeta=\mathcal{F}^{-1}(\sqrt{\mathcal{F}(C)} \mathcal{F}(z))$. The estimation of the average can be carried out, using the probability 427

density associated with random variable $\zeta$ (with average 0 and unit variance):

$$
\langle\exp (\omega \sigma \zeta)\rangle=\int_{\mathbb{R}} e^{\omega \sigma \zeta}\left(\frac{1}{N} e^{-\zeta^{2} / 2}\right) d \zeta,
$$

${ }_{428}$ where the normalization parameter $N$ ensures that $\int_{\mathbb{R}} \frac{1}{N} e^{-x^{2} / 2} d x=1$. 
We then obtain

$$
\begin{aligned}
\langle\exp (\omega \sigma \zeta)\rangle & =\int_{\mathbb{R}} \frac{1}{N} e^{\omega \sigma \zeta-\zeta^{2} / 2} d \zeta \\
& =\int_{\mathbb{R}} \frac{1}{N} e^{\left[(\omega \sigma)^{2}-(\zeta-\omega \sigma)^{2}\right] / 2} d \zeta \\
& =e^{(\omega \sigma)^{2} / 2} \int_{\mathbb{R}} \frac{1}{N} e^{-(\zeta-\omega \sigma)^{2} / 2} d \zeta \\
& =e^{(\omega \sigma)^{2} / 2} \int_{\mathbb{R}} \frac{1}{N} e^{-Y^{2} / 2} d Y(\text { with } Y=\zeta-\omega \sigma) \\
& =e^{(\omega \sigma)^{2} / 2},
\end{aligned}
$$

which leads to Eq. (36). A direct application of previous calculations shows that

$$
\begin{aligned}
& \langle\eta\rangle^{2}=\eta_{g}^{2} \exp \left(\sigma^{2}\right), \\
& \left\langle\eta^{2}\right\rangle=\eta_{g}^{2} \exp \left(2 \sigma^{2}\right) .
\end{aligned}
$$

The variance $C(0)$ of $\eta$ is then given by

$$
C(0)=\eta_{g}^{2} \exp \left(\sigma^{2}\right) \times\left[\exp \left(\sigma^{2}\right)-1\right] .
$$

For small $\log$ viscosity variance $\sigma^{2}$, one obtains a direct relation between the variance of viscosity and the variance of the corresponding logarithm:

$$
C(0) \simeq \eta_{g}^{2} \sigma^{2}
$$

[1] A. Einstein, Investigations on the Theory of the Brownian Movement (Dover Publications, New York, 1956).

[2] G. I. Taylor, The viscosity of a fluid containing small drops of another fluid, Proc. R. Soc. Lond. A 138, 41 (1932).

[3] G. Batchelor, The stress system in a suspension of force-free particles, J. Fluid Mech. 41, 545 (1970).

[4] J. F. Brady and G. Bossis, Stokesian dynamics, Annu. Rev. Fluid Mech. 20, 111 (1988).

[5] J. F. Brady, The rheological behavior of concentrated colloidal dispersions, J. Chem. Phys. 99, 567 (1993).

[6] K. D. Danov, On the viscosity of dilute emulsions, J. Colloid Interface Sci. 235, 144 (2001).

[7] J. D. Williams, W. Svrcek, and W. Monnery, The prediction of viscosity for mixtures using a modified square well intermolecular potential model, Dev. Chem. Eng. Miner. Process. 11, 267 (2003).

[8] E. Koval et al., A method for predicting the performance of unstable miscible displacement in heterogeneous media, Soc. Petrol. Eng. J. 3, 145 (1963).

[9] R. J. Blackwell, J. R. Rayne, and W. M. Terry, Factors influencing the efficiency of miscible displacement, Trans. AIME 216, 1 (1959).

[10] L. D. Landau and E. M. Lifshitz, Electrodynamics of Continuous Media, Volume 8 of A Course of Theoretical Physics (Pergamon Press, 1960).

[11] G. Matheron, Eléments pour une théorie des milieux poreux (Masson, Paris, 1967) .

[12] G. Dagan, Flow and Transport in Porous Formations (Springer-Verlag, Berlin, Heidelberg, New York, 1989).

[13] B. Noetinger and Y. Gautier, Use of the Fourier-Laplace transform and of diagrammatical methods to interpret pumping tests in heterogeneous reservoirs, Adv. Water Resour. 21, 581 (1998).

[14] Y. A. Stepanyants and E. V. Teodorovich, Effective hydraulic conductivity of a randomly heterogeneous porous medium, Water Resour. Res. 39, 1065 (2003). 
[15] F. Willot and D. Jeulin, Elastic behavior of composites containing boolean random sets of inhomogeneities, Int. J. Eng. Sci. 47, 313 (2009).

[16] V. V. Jikov, S. M. Kozlov, and O. A. Oleinik, Homogenization of Differential Operators and Integral Functionals (Springer Science \& Business Media, Berlin, Heidelberg, New York, 2012).

[17] B. Noetinger, The effective permeability of a heterogeneous porous medium, Transp. Porous Media 15, 99 (1994).

[18] A. De Wit, Correlation structure dependence of the effective permeability of heterogeneous porous media, Phys. Fluids 7, 2553 (1995).

[19] P. Renard and G. De Marsily, Calculating equivalent permeability: A review, Adv. Water Resour. 20, 253 (1997).

[20] M. Quintard and S. Whitaker, Transport in chemically and mechanically heterogeneous porous media. III. Large-scale mechanical equilibrium and the regional form of Darcy's law, Adv. Water Resour. 21, 617 (1998).

[21] L. Petit and B. Noetinger, Shear-induced structures in macroscopic dispersions, Rheol. Acta 27, 437 (1988).

[22] R. Scirocco, J. Vermant, and J. Mewis, Effect of the viscoelasticity of the suspending fluid on structure formation in suspensions, J. Non-Newtonian Fluid Mech. 117, 183 (2004).

[23] A. Y. Malkin, A. Semakov, and V. Kulichikhin, Self-organization in the flow of complex fluids (colloid and polymer systems): Part 1: Experimental evidence, Adv. Colloid Interface Sci. 157, 75 (2010).

[24] R. Chatelin, D. Anne-Archard, M. Murris-Espin, M. Thiriet, and P. Poncet, Numerical and experimental investigation of mucociliary clearance breakdown in cystic fibrosis, J. Biomech. 53, 56 (2017).

[25] P. J. Mucha, S.-Y. Tee, D. A. Weitz, B. I. Shraiman, and M. P. Brenner, A model for velocity fluctuations in sedimentation, J. Fluid Mech. 501, 71 (2004).

[26] R. Romeu and B. Noetinger, Calculation of internodal transmissivities in finite difference models of flow in heterogeneous porous media, Water Resour. Res. 31, 943 (1995).

[27] M. Le Ravalec, B. Noetinger, and L. Y. Hu, The FFT moving average (FFT-MA) generator: An efficient numerical method for generating and conditioning Gaussian simulations, Math. Geol. 32, 701 (2000).

[28] C. Beenakker, The effective viscosity of a concentrated suspension of spheres (and its relation to diffusion), Physica A 128, 48 (1984).

[29] B. Noetinger, A two fluid model for sedimentation phenomena, Physica A 157, 1139 (1989).

[30] S. P. Neuman and S. Orr, Prediction of steady state flow in nonuniform geologic media by conditional moments: Exact nonlocal formalism, effective conductivities, and weak approximation, Water Resour. Res. 29, 341 (1993).

[31] P. Indelman and B. Abramovich, A higher-order approximation to effective conductivity in media of anisotropic random structure, Water Resour. Res. 30, 1857 (1994).

[32] B. Nœtinger, Computing the effective permeability of log-normal permeability fields using renormalization methods, Comptes Rendus de l'Académie des Sciences-Series IIA-Earth and Planetary Science 331, 353 (2000).

[33] B. Abramovich and P. Indelman, Effective permittivity of log-normal isotropic random media, J. Phys. A 28, 693 (1995).

[34] R. Chatelin and P. Poncet, A hybrid grid-particle method for moving bodies in a 3D Stokes flow with variable viscosity, SIAM J. Sci. Comput. 35, B925 (2013).

[35] R. Chatelin and P. Poncet, Hybrid grid-particle methods and penalization: A Sherman-MorrisonWoodbury approach to compute 3D viscous flows using FFT, J. Comput. Phys. 269, 314 (2014).

[36] R. Chatelin and P. Poncet, A parametric study of mucociliary transport by numerical simulations of 3D non-homogeneous mucus, J. Biomech. 49, 1772 (2016). 